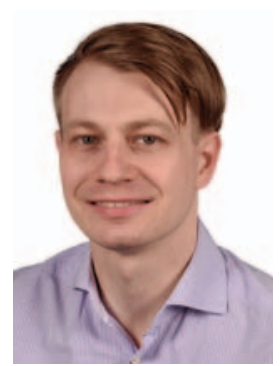

Dr. Sebastian Zimmer

Koordination «DermaCampus»

\section{Meilensteine auf dem Weg zum Arztdasein: Zeitmanagement - Promotion - Diagnosestellung}

Auch die aktuelle Ausgabe des «DermaCampus» beleuchtet viele Meilensteine auf dem Weg zum Arztdasein. Zu Beginn werfen wir Sie gleich in das vertraute kalte Wasser und erinnern Sie in der Fortsetzung unserer Serie zu Differentialdiagnosen an die Hürden der Diagnose einer Hidradenitis suppurativa (HS). Diese Erkrankung ist bei einer hohen Dunkelziffer an Betroffenen nicht diagnostiziert, obwohl die HS an sich nicht schwer zu deuten ist. Mit diesem Beitrag von Frau Dr. Sophia Wilden von der Hautklinik Mainz möchten wir das Bewusstsein für die Möglichkeit einer frühen Diagnose und Therapie schärfen, sodass damit auch Komorbiditäten dieser Erkrankung minimiert werden könnten. Eine frühzeitige Diagnosestellung ist jedoch nur die halbe Miete: Wie sage ich es meinem Patienten? Das Schauspielpatienten-Programm an der Medizinischen Fakultät der Universität Würzburg unterstützt Medizinstudierende schon frühzeitig beim Erlernen dieser Kunst. Bevor Sie jedoch in der Praxis aktiv werden, tut sich vielleicht die Frage auf: Promotion - ja oder nein? Professor Axel W. Bauer steht dazu im Rahmen unserer Reihe zum wissenschaftlichen Schreiben und Publizieren Rede und Antwort. Ein weiteres grundlegendes Thema während des Arztdaseins ist der Spagat zwischen Karriere und Familie. Prof. Dorothee Nashan, Vorsitzende der Arbeitsgemeinschaft Frauen in der Dermatologie, hat viele hilfreiche Tipps dazu. Clara Vollmers Buchrezension zu Michael Sabels «Time and Life Management for Medical Students and Residents» rundet diesen Themenkomplex und schließt den «DermaCampus» dieser Ausgabe ab.

Haben Sie viel Freude beim Lesen! Mit besten Grüßen

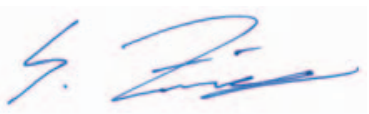

\section{KARGER}

() 2017 S. Karger GmbH, Freiburg

Fax +497614520714

information@karger.com www.karger.com
Dr. Sebastian Zimmer

Universitätsmedizin der Johannes Gutenberg

Universität Mainz

Hautklinik und Poliklinik, Geb. 401, CRC

Langenbeckstr. 1, 55131 Mainz, Deutschland 
Die Hidradenitis suppurativa oder auch Acne inversa hat mit der Zulassung von Adalimumab im Jahr 2015 neue Aufmerksamkeit erhalten. Trotz des Fokus auf diese Erkrankung bleibt die Dunkelziffer der Betroffenen hoch. Zu den Gründen hierfür gehört schlicht ein Nicht-Abfragen von Symptomen, wenn sich ein Patient zur Spaltung eines akuten Abszesses vorstellt. Zudem kommt es vor, dass die Diagnose trotz re- zidivierender Abszesse in den großen Körperfalten nicht gestellt wird. Man sollte sich bewusst machen, dass es sich bei der Acne inversa, wie bei Psoriasis, um eine der sogenannten Immune mediated inflammatory disorders (IMID) handelt [1]. Dabei ist es sehr wichtig, die Diagnose so früh wie möglich zu stellen, um den Progress dieser mutilierenden, systemischen Erkrankung zu verhindern.

\section{? Wichtige Fragen}

\section{Was ist das Problem?}

Das große Problem der späten Diagnosestellung, welche im Schnitt erst 7 Jahre nach dem ersten Auftreten gestellt wird, ist die Entwicklung irreversibler Narben- und Fistelbildung. Die Erkrankung wird gemäß ihrer Schwere in die sogenannten HurleyStadien eingeteilt (Tab. 1) [2]. Allgemein bekannt ist die Tatsache, dass die Patienten häufig übergewichtig und nikotinabhängig sind. Beide Faktoren scheinen in der Genese eine Rolle zu spielen, jedoch gibt es bisher keine Daten, die zeigen, dass eine Änderung dieser Faktoren zu einer deutlichen Besserung führen würde [3]. Häufige Komorbidität ist das metabolische Syndrom (wobei die arterielle Hypertonie nicht gehäuft aufzutreten scheint), das polyzystische Ovarsyndrom, chronisch-entzündliche Darmerkrankungen, aber auch Spondylarthropathien [4]. Die Behandlung der Erkrankung ist schwierig. Je weiter sie fortschreitet, desto unumgänglicher ist in vielen Fällen eine operative Sanierung, vor welcher sich viele Patienten aufgrund der oftmals großen Wunden, welche optimalerweise sekundär heilen sollten, fürchten. Dass die HS in vielen Fällen mit erhöhten Zytokinleveln (TNF-a, IL-1, IL-17), aber auch dauerhaft erhöhten CRP- und Leukozytenwerten einhergeht, macht deutlich, wie hoch die systemische Entzündungslast bei dieser Erkrankung ist [5].
Tab. 1 Hurley-Klassifikation

\begin{tabular}{|c|c|}
\hline $\begin{array}{l}\text { Hurley } \\
\text { Klassifikation }\end{array}$ & Klinische Präsentation \\
\hline Grad I & $\begin{array}{l}\text { Einzelne Abszesse, keine Fistelgänge, } \\
\text { keine Vernarbungen }\end{array}$ \\
\hline Grad II & $\begin{array}{l}\text { Ein oder mehrere auseinander liegende Abszesse, } \\
\text { mit Fistelgängen und Narbenbildung }\end{array}$ \\
\hline Grad III & $\begin{array}{l}\text { Flächiger Befall mit Abszessen, Fistelgängen und } \\
\text { Narbenzügen }\end{array}$ \\
\hline
\end{tabular}

\section{Was ist die Lösung?}

Aufgrund der begrenzten Behandlungsmöglichkeiten, ist es umso wichtiger, die Diagnose frühzeitig zu stellen, die Patienten aufzuklären und nach Komorbiditäten zu screenen. Die Diagnose HS sollte gestellt werden, sobald die Patienten innerhalb von 6 Monaten mehr als zweimal unter schmerzhaften Knoten oder Abszessen leiden, welche axillär, inguinal, perigenital/perineal, gluteal oder auch submammär lokalisiert sind [6].

\section{Therapie}

Wie bei den anderen chronisch-entzündlichen Hauterkrankungen kann man die Therapie in eine Basistherapie und eine eskalierende Stufentherapie einteilen. Da Nikotin und Körperfett ein proinflammatorisches Milieu generieren, welches die Wahrscheinlichkeit und das Fortschreiten letztlich aller chronisch-entzündlichen Erkrankungen fördert, sollte über diese Risikofaktoren aufgeklärt werden [7]. Es wird außerdem empfohlen, auf eine Nassrasur in den betroffenen Arealen zu verzichten, da die Mikrotraumata im Sinne einer Köbnerisierung wie bei Psoriasis weiterer Trigger sein können. Gleiches gilt für das Tragen enger, zu Reibung führender Kleidung [8]. Basistherapeutisch kann der Einsatz von antiseptischen Duschgels in den betroffenen Arealen erfolgen. Im Schub erfolgt die Therapie stadienabhängig (siehe Schaubild). Grund- pfeiler in allen Stadien der Therapie bleibt die chirurgische Behandlungsoption. Bei beginnenden entzündlichen Knoten kann eine lokale Antibiose mit 1\%iger Clindamycinlösung aber gegebenenfalls auch ausreichend sein. Nächster Schritt der Stufentherapie wäre die systemische Antibiose. Welche in frühen Stadien (Hurley-Stadium I oder frühes Stadium II) aus Tetracyclin 500 mg zweimal täglich über bis zu 4 Monate, in späteren Stadien aus Clindamycin 300 mg zweimal täglich und Rifampicin 600 mg einmal täglich über 8-12 Wochen besteht. Bei Nicht-Ansprechen auf die obengenannte Therapie folgt der Einsatz von Adalimumab mit einer Erhaltungsdosis von 40 mg wöchentlich [9]. Die gute Entzündungskontrolle kann das Ausmaß des chirurgischen Interventionsbedarfs reduzieren [10]. 


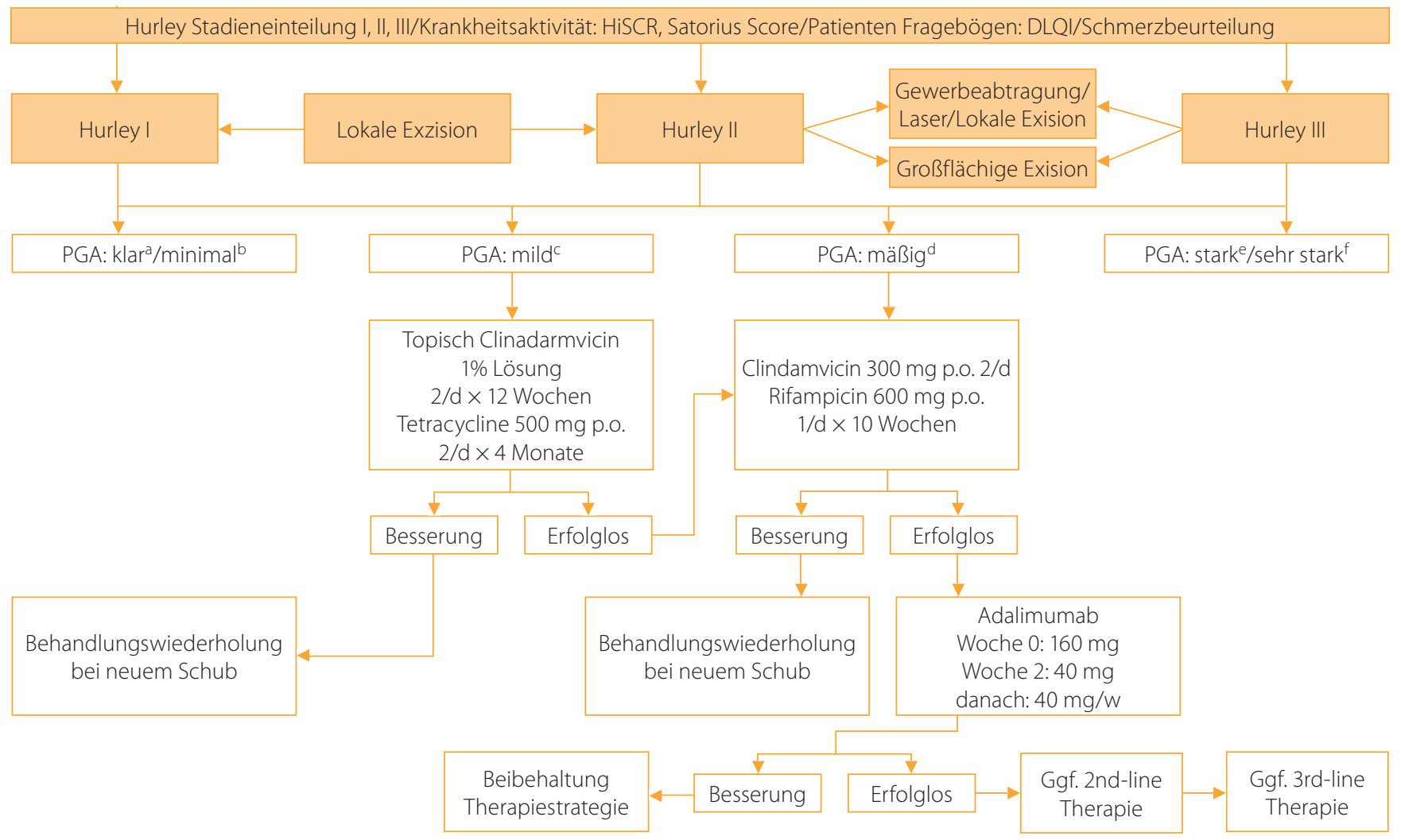

Behandlungspfad gemäß der europäischen Hidradenitis suppurativa-Leitlinie. PGA = physician global assesment. ${ }^{2}$ Klar: keine Entzündungen oder nicht-entzündliche Knoten. bMinimal: bis zu 5 nicht-entzündliche Knoten. 'Mild: weniger als 5 entzündliche Knoten oder ein Abszess oder eine drainierende Fistel und keine entzündlichen Knoten. ¿Mäßig: weniger als 5 entzündliche Knoten oder ein Abszess; oder eine drainierende Fistel und ein oder mehr entzündliche Knoten; oder 2-5 Abszesse oder drainierende

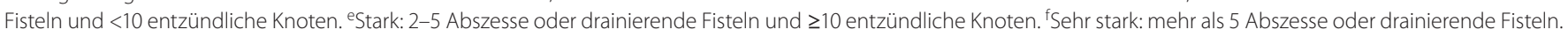

\section{Literatur}

1 Kelly G, Prens EP: Inflammatory mechanisms in hidradenitis suppurativa. Dermatol Clin 2016;34:51-58.

2 Zouboulis CC, Bechara FG, Fritz K, et al.: S1-Leitlinie zur Therapie der Hidradenitis suppurativa/Acne inversa, AWMF Register-Nummer 013-012. www.awmf.org/ uploads/tx_szleitlinien/013-012l_S1_Acne_inversa_Hidradenitis_suppurativa_ 2012-12.pdf (letzter Zugriff 08.09.2017)

3 Cesko E, Koerber A, Dissemond J: Smoking and obesity are associated factors in acne inversa: results of a retrospective investigation in 100 patients. Eur J Dermatol 2009;19:490-493.

4 Porter ML, Kimball AB: Comorbidities of hidradenitis suppurativa. Semin Cutan Med Surg 2017;36:55-57.

5 van der Zee HH, de Ruiter L, van den Broecke DG, et al.: Elevated levels of tumour necrosis factor (TNF)- $\alpha$, interleukin (IL)- $1 \beta$ and IL-10 in hidradenitis suppurativa skin: a rationale for targeting TNF- $\alpha$ and IL-1 $\beta$. Br J Dermatol 2011;164:1292-1298.

6 Miller JM, Ellervik C, Vinding GR, et al.: Association of metabolic syndrome and hidratenitis suppurativa. JAMA Dermatol 2014;150:1273-1280.
7 Miller IM, Ellervik C, Vinding GR, et al.: Association of metabolic syndrome and hidradenitis suppurativa. JAMA Dermatol 2014;150:1273-1280.

8 Dufour DN, Bryld LE, Jemec GB: Hidradenitis suppurativa complicating naevus comedonicus: The possible influence of mechanical stress on the development of hidradenitis suppurativa. Dermatology 2010;220:323-325.

9 Gulliver W, Zouboulis C, Prens E, et al.: Evidence-based approach to the treatment of hidradenitis suppurativa/acne inversa, based on the European guidelines for hidradenitis suppurativa. Rev Endocr Metab Disord 2016;17:343-351.

10 Falola RA, DeFazio MV, Anghel EL, et al.: What heals hidradenitis suppurativa: Surgery, immunosuppression, or both? Plast Reconstr Surg 2016;138:219S-229S.

Kontaktadresse: Dr. Sophia Wilden, Universitätsmedizin der Johannes Gutenberg Universität Mainz, Hautklinik und Poliklinik, Gebäude 401, Langenbeckstraße 1, 55131 Mainz, Deutschland, sophia.wilden@unimedizin-mainz.de. 
Arzt-Patienten-Kommunikation

Schauspielpatienten-Programm an der Medizinischen Fakultät der Universitåt Würzburg

Krankheitsverlauf, Adhärenz, Behandlungserfolg und nicht zuletzt eine gelungene Kommunikation [1] sind Aspekte, die eine gute ArztPatienten-Beziehung positiv beeinflussen. Um den Grundstein für diese essenziellen kommunikativen Fähigkeiten möglichst bereits im Medizinstudium zu legen, werden weltweit an zahlreichen Universitäten Schauspielpatienten eingesetzt, d.h. Laienschauspieler, die für bestimmte Patientenrollen trainiert werden: Dies umfasst nicht nur eine medizinische Vorgeschichte mit Symptomen, Voruntersuchungen usw., sondern auch nicht-medizinische Informationen wie den Beruf oder die familiäre Situation des erdachten Patienten. Ziel ist es, einen möglichst authentischen Patienten entstehen zu lassen. Seit 2007 gibt es das Schauspielpatienten-Programm an der Universität Würzburg. Wir hatten die Möglichkeit, mit Dr. Matthias Lukasczik und Dipl.Psych. Hans-Dieter Wolf, den beiden Koordinatoren des Programms, zu sprechen.

\section{Wie läuft ein Schauspielpatientengespräch ab?}

Die Gespräche finden in Kleingruppen von maximal 12 Personen statt und dauern jeweils ca. 10 min. Sie werden in den Lehrveranstaltungen vorbereitet, d.h. die Dozenten erarbeiten mit den Studenten allgemeine Informationen und geben Input zur Situation und zu den Fertigkeiten, die für ein gutes Gespräch wichtig sind. Außerdem erhalten die Studenten eine schriftliche Kurzinformation mit Angaben zum Patienten, zu medizinischen Fakten und zu ihrer Aufgabe als Arzt im Gespräch.

Im Gespräch selbst sind jeweils ein Dozent bzw. Tutor sowie die anderen Mitglieder der Kleingruppe anwesend. Nach dem Gespräch folgt ein strukturiertes Feedback: Nachdem der Student, der die Arztrolle übernommen hat, seine Einschätzung des Gesprächs abgegeben hat, äußert sich der Schauspielpatient (SP) dazu, was im

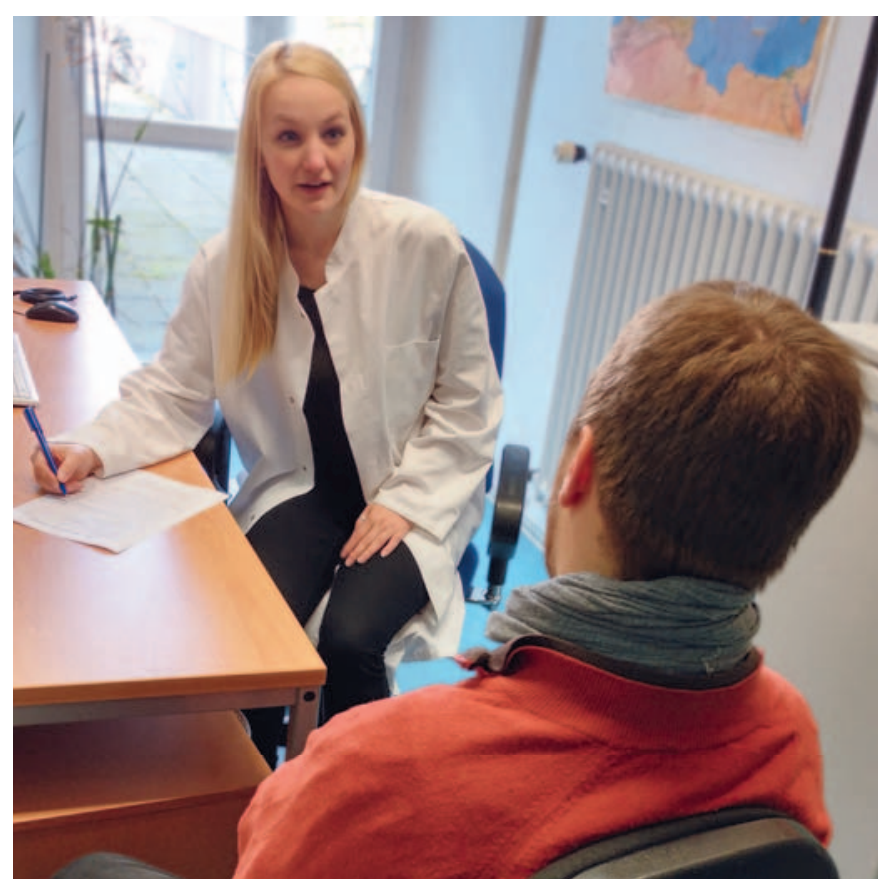

${ }^{\circledR}$ Matthias Lukasczik, Schauspielpatienten-Programm Würzburg.
Gespräch gut gelaufen ist und an welchen Stellen es Verbesserungsbedarf gibt. Danach geben der Dozent und die anderen Kursteilnehmer Rückmeldung.

\section{Wie wird eine Patientenrolle kreiert?}

Zunächst überlegen wir uns, wo Bedarffür eine neue Rolle besteht. Hieraus leiten sich in der Regel schon einige Vorgaben ab (Erkrankung, Lebensstil des Patienten, vermutliche Gefühlslage usw.).

Mithilfe dieses Grundgerüstes folgt in der Regel zuerst eine Art Brainstorming mit den für das jeweilige Fach verantwortlichen Dozenten. An der detaillierten Formulierung der Rolle sind immer mehrere Personen aus verschiedenen Berufsgruppen (Psychologen, Ärzte) beteiligt.

Die Rolle umfasst im Ergebnis dann mindestens die folgenden Informationen:

- Angaben zur Person (Name, Alter inkl. Geburtsdatum, Familie)

- Angaben zur beruflichen Situation und Laufbahn

- Angaben zur aktuellen medizinischen Situation

- Angaben zur medizinischen Vorgeschichte

- Angaben zu Persönlichkeit und sozialer Situation

Die so entstandene Rolle wird dann mit einem oder mehreren SP durchgearbeitet und im Rollentraining praktisch getestet. Input von Schauspielerseite wird aufgegriffen, mögliche Unklarheiten oder «Stolpersteine» werden berücksichtigt und die Rolle entsprechend angepasst. Erst dann kann sie in der Lehrroutine eingesetzt werden.

Gibt es «schwierige» Rollen, die beispielsweise den kulturellen Hintergrund und die damit verbundenen Herausforderungen berücksichtigen?

Es gibt Rollen, die aufgrund ihrer medizinischen Situation herausfordernd sind - dazu zählt z.B. die Rolle einer jungen Mutter mit fortgeschrittener Krebserkrankung, die aller Wahrscheinlichkeit nach ihr Kind nicht aufwachsen sehen wird. Andere sicher sinnvolle und spannende Konstellationen, z.B. Angehörigengespräche oder Rollen aus anderen kulturellen Hintergründen, sind Teil unserer langfristigen Planungen, bislang aber noch nicht umgesetzt.

Welche Problemfelder/Herausforderungen haben sich im Laufe der Jahre herauskristallisiert und was kann man daraus für die Praxis lernen?

Eine der größten Schwierigkeiten, mit der gerade junge Mediziner zurechtkommen müssen, ist der immer größer werdende Zeitdruck im ärztlichen Berufsalltag. Eine der großen Herausforderungen ist es daher, zu zeigen, dass man auch in zehn Minuten eine gute Beziehung zu einem Patienten aufbauen und auf seine Bedürfnisse eingehen kann.

Aufgrund der demographischen Entwicklung nimmt auch die Arbeit mit älteren Menschen einen immer größeren Raum in der ärztlichen Arbeit ein. Deshalb sind mehrere Rollen im Würzburger SP- 
Programm bereits auf ältere Patienten zugeschnitten, um den Studierenden das Üben alltagsrelevanter Gesprächssituationen zu ermöglichen.

Welche Entwicklungen gab es in den nunmehr 10 Jahren, in denen das Programm schon läuft und welche Pläne gibt es für die Weiterentwicklung des Programms?

In erster Linie ist das Programm ausgebaut und erweitert worden - sowohl was den Pool an Rollen als auch was die Zahl der Lehrveranstaltungen mit SP-Einsätzen betrifft. Mittlerweile ist die Arbeit mit SP ein fester Bestandteil der medizinischen Lehre in Würzburg und wird von den Studenten sehr gut bewertet. Der hohe Zuspruch zeigt sich auch an den vielen Interessierten, die sich als Tutoren bzw. als SP bewerben. Die gute Entwicklung des Programms findet fächerübergreifend Anerkennung und wurde 2011 mit dem AlbertKölliker-Lehrpreis der Medizinischen Fakultät ausgezeichnet.

Ein Faktor, der für die medizinische Ausbildung sicher relevant ist, ist der Nationale Kompetenzbasierte Lernzielkatalog Medizin (NKLM). Dort wird Kommunikationsfertigkeiten ein großer Stellenwert beigemessen. Daher wird sicher die Frage nach der Prüfbarkeit der erlernten Kommunikationskompetenzen, z.B. im Rahmen von OSCE-Prüfungen, noch mehr in den Vordergrund rücken. Hier steht das SP-Programm in Würzburg noch in der Planungsphase.

\section{Wir danken Ihnen herzlich für das Interivew!}

\section{Angaben zum Schauspielpatienten-Programm in Würzburg}

Das Programm wurde 2007 von Ärzten und Psychologen des Uniklinikums (Palliativmedizin) und der Universität (Medizinische Psychologie) initiiert, damals noch beschränkt auf den Bereich Onkologie. Aktuell werden Schauspielpatienten in vier Lehrveranstaltungen eingesetzt: «Medizinische Psychologie» (Anamnese, Motivierung zur Lebensstiländerung, Aufklärung/ Diagnosemitteilung), «Praktische Klinische Untersuchungsmethoden» (Anamnese), «Kommunikationstraining Interdisziplinäre Onkologie» (Diagnosemitteilung) und «OP-Aufklärung». Insgesamt kommen derzeit ca. 30 Schauspielerinnen und Schauspieler in 20 verschiedenen Rollen zum Einsatz.

Nähere Informationen finden Sie unter www.psychotherapie.uni-wuerzburg.de/schauspielpatienten

\author{
Literatur \\ 1 Interview mit Samy Molcho: Von Mensch zu Mensch statt von Arzt zu Patient. \\ Karger Kompass Dermatol 2017:5;98.
}

Kontaktadresse: Dr. Matthias Lukasczik / Dipl.-Psych. Hans-Dieter Wolf, JuliusMaximilians-Universität Würzburg, Abteilung für Medizinische Psychologie, Klinikstraße 3, 97070 Würzburg, Deutschland, matthias.lukasczik@ uniwuerzburg.de; hans.wolf@uni-wuerzburg.de.

\section{Interview mit Prof. Axel W. Bauer \\ Über die medizinische Promotion}

Diemeisten Medizinstudierenden wollen promovieren, doch wiegeht das? Wie findet man das passende Thema, den richtigen Betreuer und wann beginnt man am besten mit derPlanung? Prof.AxelW. Bauerhat an einem Ratgeber für Mediziner mitgeschrieben, die promovieren möchten. Im Interview erklärt er, wie man die eigene Promotion am besten plant und wann man das Promotionsprojekt besser als erledigt betrachten sollte.

Herr Prof. Bauer, mit welcher Problematik haben angehende Mediziner bei ihrer Promotion besonders zu kämpfen?

Jedes Kapitel unseres Ratgebers beschreibt einen wichtigen Punkt, aber ganz wesentlich erscheint mir die Frage des Zeitpunkts und der Themensuche. Denn wenn man das falsche Thema erwischt hat oder den falschen Zeitpunkt, dann wird die Sache nicht glücken.

\section{Wann ist denn der richtige Zeitpunkt, um mit der Doktorarbeit zu beginnen?}

Man sagt einerseits: nicht zu früh. Also möglichst nicht schon im Grundstudium. Ausgeschlossen ist das zwar nicht, aber der günstigere Zeitpunkt ist sicher eher in den ersten Semestern des Hauptstudiums, also im 3. oder 4. Studienjahr, wobei dann wiederum das Problem hinzukommt, dass man möglichst nach dem 5. Studienjahr fertig sein möchte. Die Zeit kann dann knapp werden, und dann zieht sich die Bearbeitung der Dissertation über das Staatsexamen hinaus.
Eine Doktorarbeit während der Weiterbildung zu verfassen halten Sie also für schwierig?

Das ist ohne Zweifel sehr schwierig. Immer wieder melden sich auch bei mir Kandidaten in diesem Berufsstadium, aber sie unterschätzen, wie viel Arbeit eine Dissertation macht. Man muss enorm viel Mühe investieren, um Bibliotheken aufzusuchen oder auch mal ein Archiv, oder um Experimente zu machen. Wenn man abends um 19 Uhr müde nach Hause kommt, dann verschiebt man das Forschen aufs Wochenende, da braucht man aber eher Erholung. In der Weiterbildungsphase die Doktorarbeit zu schreiben ist also nicht sehr günstig, auch wenn es auch hier hin und wieder den für mich erstaunlichen Fall gibt, dass es jemand schafft. Aber es ist eher selten, dass das gelingt.

\section{Wie findet man das richtige Thema für seine Doktorarbeit?}

Ich hatte eine angehende Doktorandin zu Besuch, die habe ich das auch gefragt. Sie sagte mir, unter ihren Kommilitonen gelte es als ganz besonders wichtig, wer Doktorvater oder Doktormutter wird, der oder die sich dann auch um das Thema und seine Betreuung kümmert. Die Persönlichkeit des Betreuers spielt bei der Themenwahl also offenkundig eine überragende Rolle. Dann geht es natürlich auch noch um die Frage: Für was für eine Art von Doktorarbeit eigne ich mich? Es beginnt mit retrospektiven klinischen Studien, bei denen schon vorhandenes Datenmaterial mit fragli- 
cher Qualität der Daten analysiert wird. Diese Arbeiten gelingen in der Regel, denn sie sind relativ leicht zu bewältigen, allerdings auch wenig spannend, da man hier ja keine Hypothese beweisen, sondern allenfalls eine Hypothese generieren kann.

Anders ist es mit klinisch-prospektiven oder mit epidemiologischen Arbeiten, bei denen man die Daten erst gewinnen muss. Das kann länger dauern als man zunächst glaubt. Ähnlich ist es bei einer experimentellen Arbeit. Nur bei einer prospektiven und experimentellen Arbeit ist es möglich, eine These zu bestätigen oder zu entkräften. Das gilt als wissenschaftlich wertvoller, wird dann auch mit besseren Noten wie «magna cum laude» oder «summa cum laude» belohnt. Solche Arbeiten haben aber auch das Risiko, dass sie scheitern, weil nicht genügend Patienten zur Untersuchung vorhanden sind, der Beobachtungszeitraum zu kurz ist oder sich die Hypothese nicht bestätigen lässt.

Schließlich gibt es auch noch «Literaturarbeiten», bei denen man auch eine sehr gute Note bekommen kann. Allerdings sind diese Arbeiten - besonders in meinem Fachgebiet Geschichte, Theorie und Ethik der Medizin - methodisch vom naturwissenschaftlich dominierten Medizinstudium recht weit entfernt, sodass es dann auch nicht schadet, wenn jemand sprachliche, philosophische, juristische oder theologische Interessen beziehungsweise Vorkenntnisse hat.

Man sollte sich also zunächst einmal überlegen, wo die eigene Neigung hingeht, und dann auch eruieren, was an der Medizinischen Fakultät angeboten wird. Viele Betreuer haben eine aussagekräftige Homepage, auf der man erkennen kann, was da früher schon an Doktorarbeiten

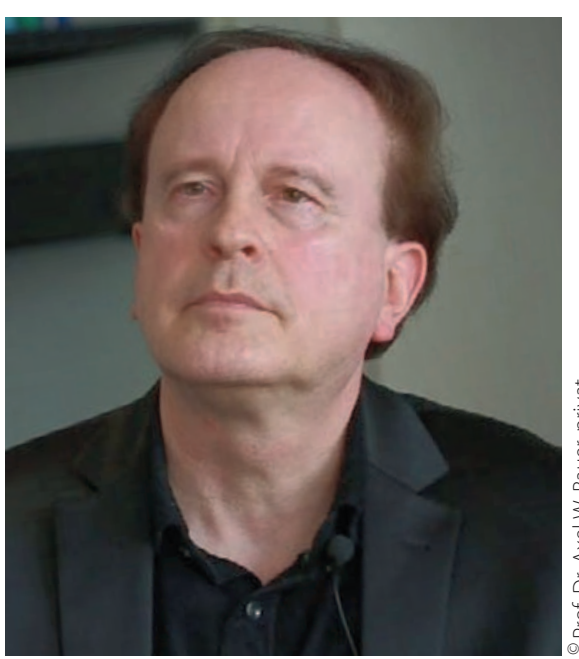

«Promotion macht Sinn aber nicht um jeden Preis»
Kommen wir zu den praktischen Tipps: Ist es sinnvoll die Literatur

Wenn man beginnt, Literatur zu sammeln, ist es wichtig, erst einmal zu schauen, wie die entsprechenden Zitierregeln an der jeweiligen Fakultät sind. Das heißt, bestimmte Zitierweisen werden an einer Medizinischen Fakultät vorgeschrieben, während an einer anderen Fakultät wieder ganz andere Regeln verpflichtend sind. An diese Regeln sollte man sich von Anfang an halten, gleichgültig, ob man ein Literaturverwaltungsprogramm verwendet wie EndNote oder Citavi oder wie sie alle heißen mögen.

Man kann auch ohne ein Literaturverwaltungsprogramm arbeiten. Ich selbst z.B. mache meine Literaturverwaltung grundsätzlich von Hand, und das, obwohl gerade ein Medizinhistoriker und Medizinethiker sehr viel Literatur kennen und zitieren muss. Unabhängig davon, wie man die gelesene Literatur verwaltet, sollte man das mit einem Literaturverwaltungsprogramm zu katalogisieren? Gelesene von Anfang an gut dokumentieren, weil man ja doch unterschätzt, was man alles wieder vergisst. Wenn man sich von Anfang an Notizen gemacht und die Literatur gesammelt hat, dann hat man es später leichter.

Gibt es an den Universitäten Hilfestellung jenseits der betreuenden Professoren?

Ja, die gibt es, z.B. bei uns an der Medizinischen Fakultät Mannheim der Universität Heidelberg. Wir haben seit dem Wintersemester 2015/16 im 3. Studienjahr den obligatorischen Leistungsnachweis «Wissenschaftliches Arbeiten». Diesen Leistungsnachweis müssen alle Studierenden erbringen. Zum Abschluss müssen die Studierenden - spätestens bis zum Ende des 5 . Studienjahres - eine Forschungsarbeit von betreut worden ist, wie die Themen lauten und was die Forschungsschwerpunkte sind. Man sollte sich auf jeden Fall kundig machen, bevor man den direkten Kontakt sucht, weil Doktorväter und -mütter es natürlich gerne sehen, wenn ein potenzieller Doktorand eine gewisse Vorstellung hat, was in dem entsprechenden Bereich, in der Abteilung oder in der Klinik geforscht wird.

Ist es notwendig, die Promotion in dem Bereich zu machen, in dem später auch die Weiterbildung absolviert wird?

Nein, sicher nicht. Ich selbst, der ich heute Medizinhistoriker, Wissenschaftsphilosoph und Medizinethiker bin, habe 1979 in der Hals-Nasen-Ohren-Klinik in Freiburg über die stereophone Hörgeräteversorgung promoviert. Oder denken Sie an die Fächer des Grundstudiums, also Anatomie, Physiologie und Biochemie. Da promovieren nicht nur Kollegen, die später Physiologe oder Biochemiker werden wollen. Es ist durchaus möglich, in dem Fach, auf das man sich später spezialisiert, bereits die Dissertation zu schreiben. Aber die Promotion ist keine Vorentscheidung in dem Sinn, dass man danach nichts anderes mehr machen könnte. etwa 15 Seiten schreiben. Das ist eine Vorstufe zur Doktorarbeit, da kann man sich schon den Betreuer aussuchen, bei dem man später vielleicht promovieren will und fertigt unter seiner Leitung eine Forschungsarbeit an. Diese Arbeit wird vom Betreuer in 7 Dimensionen benotet, und aus den besten Absolventen wählt man als künftiger Doktorvater seine Doktoranden leichter aus als früher. Das hat sich bei uns in den letzten 2 Jahren schon sehr bewährt, denn dadurch bekommt die Wissenschaftlichkeit schon im Hauptstudium einen hohen Wert.

Die Promotion ist keine Pflicht. Wann würden Sie einem Studierenden, der zu Ihnen mit einem Promotionswunsch kommt, von dem Schreiben einer Dissertation abraten?

Da haben wir in Mannheim fast schon ideale Verhältnisse, dass nämlich alle Studierenden eine Forschungsarbeit anfertigen müssen. Wenn jemand bei mir bei der Forschungsarbeit schon sehr gut ist, kann er danach eine Doktorarbeit übernehmen. Wenn jemand bei der Forschungsarbeit aber zu der Erkenntnis kommt, dass es ihm keinen Spaß gemacht hat oder dass sie ihm nicht gut gelungen ist, dass eher die Notwendigkeit als der Wunsch nach einer wissen- 
schaftlichen Arbeit zielführend war, dann würde ich eher abraten. Denn nichts wäre frustrierender, als wenn man sich 2, 3 oder gar 4 Jahre durch eine Doktorarbeit quält, nur um den Doktorgrad zu bekommen.

Man kann nie im Voraus sicher sagen, wenn jemand eine Promotion beginnt, was dabei herauskommt. Aber ich würde sagen, etwa 20\% der Studierenden überschätzen sich und brechen ihr Vorhaben schließlich ab. Man darf nicht vergessen, sie sind meistens Anfang bis Mitte zwanzig. Da gibt es biographische Brüche, oder es wird einem eine Stelle als Assistenzärztin oder Assistenzarzt angeboten, und dann hat sie oder er keine Zeit mehr für eine Dissertation. Das alles muss man einkalkulieren.

\section{Was ist die wichtigste Charaktereigenschaft, die man benötigt, um eine Doktorarbeit erfolgreich abzuschließen?}

Selbstdisziplin ist im Berufsleben insgesamt sehr viel wichtiger, als man vielleicht in jungen Jahren meint. Dass man sich eben jeden Tag oder zu bestimmten Zeiten hinsetzt und sagt: So, jetzt schreibe ich an meiner Doktorarbeit.

Nehmen wir an, es gibt einen sehr disziplinierten Studenten, der das klare Ziel hat, die Promotion abzuschließen. Wie viel Zeit muss er dem Vorhaben einräumen?

Zunächst muss man das reguläre Studium mit dem Ziel des Staatsexamens bewältigen, sodass sich die Arbeit an der Dissertation auf die Wochenenden und die Semesterferien konzentrieren wird. Man kann sich die Daten der Semesterferien aufschreiben und einen Plan machen, doch dann kommen Prüfungen dazwischen und vieles andere.

Aber es empfiehlt sich in jedem Fall, eine bestimmte Zeit für die Doktorarbeit zu reservieren. Ob das nun jeden Tag ist, das wird man gar nicht durchhalten, denn man muss realistisch bleiben. Es gibt auch hier Zeiten, zu denen man nicht arbeiten will oder kann. Aber man kann sich vorher überlegen, wie viel Zeit bis zum Staatsexamen noch bleibt. Reicht das, um die Dissertation im Manuskript abzuschließen, oder ist das von Vornherein unrealistisch?
Was spricht Ihrer Meinung nach für einen Doktorgrad - gesellschaftliches Ansehen, besserer Verdienst?

Letzteres sicher nicht. Ist man im Krankenhaus angestellt, so bekommt man das gleiche Gehalt, unabhängig davon, ob man promoviert ist oder nicht. Allerdings, wenn man später beabsichtigt, Oberarzt oder Chefarzt zu werden, dann kommt man um eine qualifizierte Promotion nicht herum, weil die Karriere sonst rasch zu Ende geht. Denn man wird immer promovierte - und inzwischen auch qualitativ hochwertig promovierte - Kolleginnen und Kollegen haben, die dann den Vorzug bekommen. Das hohe gesellschaftliche Ansehen des Arztes ist traditionell aber schon noch ein wichtiges Motiv, denn mit dem Doktorgrad assoziiert man immer noch vor allem den Mediziner. Erst allmählich beginnt sich dieses Stereotyp zu wandeln, und das ist gut so.

\section{Zum Autor}

Prof. Dr. med. Axel W. Bauer leitet heute das Fachgebiet Geschichte, Theorie und Ethik der Medizin an der Medizinischen Fakultät Mannheim der Universität Heidelberg. Seine Promotion machte er dagegen in der Hals-Nasen-Ohren-Klinik in Freiburg. Mit dem Ratgeber «Promotion: Die medizinische Doktorarbeit - von der Themensuche bis zur Dissertation» (ed 4., Stuttgart, Thieme, 2015) wollen die Autoren Christel Weiß und Axel Bauer Studierenden der Medizin das Schreiben der Doktorarbeit erleichtern.

Kontaktadresse: Prof. Dr. med. Axel W. Bauer, Fachgebiet Geschichte, Theorie und Ethik der Medizin, Medizinische Fakultät Mannheim der Universität Heidelberg, Ludolf-Krehl-Straße 13-17, Haus C, Ebene 6, Raum 614, 68167 Mannheim, Deutschland, axel.bauer@medma.uni-heidelberg.de.

\section{Originalquelle}

Experte im Gespräch: Prof. Axel W. Bauer über die medizinische Promotion; in Operation Karriere - Das Karriereportal und der Kongress für angehende und junge Ärzte. www.operation-karriere.de. (Direktlink für Onlineversion: http://www. operation-karriere.de/karriereweg/medizinstudium/experte-im-gespraech-profaxel-w-bauer-ueber-die-medizinische-promotion.html)

\section{Familie, Arbeitszeit und Karriere}

\section{Ein Spagat ohne Dehnungsschmerz - zwischen Karriere und Familie}

\section{Selbstwirksamkeit und gute Netzwerke}

Über die chronologische Abfolge der Lebensbausteine Arbeitszeit, Familie und Karriere lässt sich streiten. Dem Studium schließen sich erste Berufsjahre an, in denen nicht selten die Familiengründung und -erweiterung eingeschlossen ist; dazu kommt immer öfter die Frage, wer von beiden Elternzeit nimmt und wenn beide, wer zuerst? Mit Blick auf die persönlichen Ambitionen und Chancen gilt es, die Berufsbiographien so weit wie möglich zu planen. Für eine realistische Selbsteinschätzung und realisierbare Visionen empfehlen sich Gespräche mit der/dem Chefin/Chef, einer/einem Mentorin/Mentor; auch schon im Rahmen jährlicher Zielgespräche im Vorfeld. Ein wesentlicher und nicht zu unterschätzender Punkt ist die eigene Profilbildung. Es gilt, eigene Stärken zu entwickeln, und der Blick auf die langfristigen Ziele darf nicht verloren gehen. Aus meiner Erfahrung bewähren sich berufliche Schwerpunkte und «Lieblingsthemen», die - auch in der Elternzeit - z.B. auf Fortbildungen und Kongressen weiterverfolgt werden können. Auch ist es sinnvoll, den Kontakt zur fördernden Klinik aufrechtzuerhalten. Damit können auch die professionellen Netzwerke erweitert werden, was hilft, sich im beruflichen Umfeld zu positionieren, bekannt und benannt zu werden, wenn es z.B. darum geht, in Gremien mitarbeiten zu dürfen.

Zahlreiche Organisationen (z.B. Marburger Bund, Ärztekammern) und Stiftungen wollen mit Blick auf die ärztliche Versorgung und mit Wertschätzung der gut ausgebildeten Ärztinnen und Ärzte helfen, unterstützen und die Zukunft entwickeln. Ein Anliegen der Ar- 
beitsgemeinschaft «Frauen in der Dermatologie» ist die Vernetzung und Beratung von Kolleginnen aber auch Kollegen für eine persönliche berufliche Weiterentwicklung. Des Weiteren gibt es Audits, welche die Familienfreundlichkeit von Kliniken prüfen (z.B. Audit «berufundfamilie» von der Hertie Stiftung), und aktive Forschungsphasen werden durch Kinderbetreuungsangebote ermöglicht (z.B. Christiane NüssleinVolhard-Stiftung).

\section{Familienfreundlichkeit im Beruf}

Als Eckpunkte für Familienfreundlichkeit im Beruf sind zwei grundlegende Voraussetzungen definiert: 1. eine an die Arbeitszeit angeglichene, sehr gute Kinderbetreuung und 2. angemessene Arbeitszeitmodelle. Für diese Basis muss flächendeckend noch mehr getan werden. Darunter fallen Arbeitszeitmodelle, die nicht auf flexibler Dominanz gründen («lch bin dann mal weg, mein Kind hat...»), sondern auf Verträgen, die verlässliche ärztliche Versorgung garantieren. Diese sollte in die Teamarbeit integrierbar und letztlich auch ökonomisch gewinnbringend sein. Für zu Hause gilt es ebenso, verlässliche Einsatzpläne von Versorgungsgaranten zu haben, um sich nicht nervös als Einzelspieler mit dem Blick auf die Uhr unter Druck zu setzen. Ein Ungleichgewicht auf beiden Seiten wird das Arbeitsverhältnis nicht langfristig erhalten, und weder die Dominanz der familiären Eigeninte-

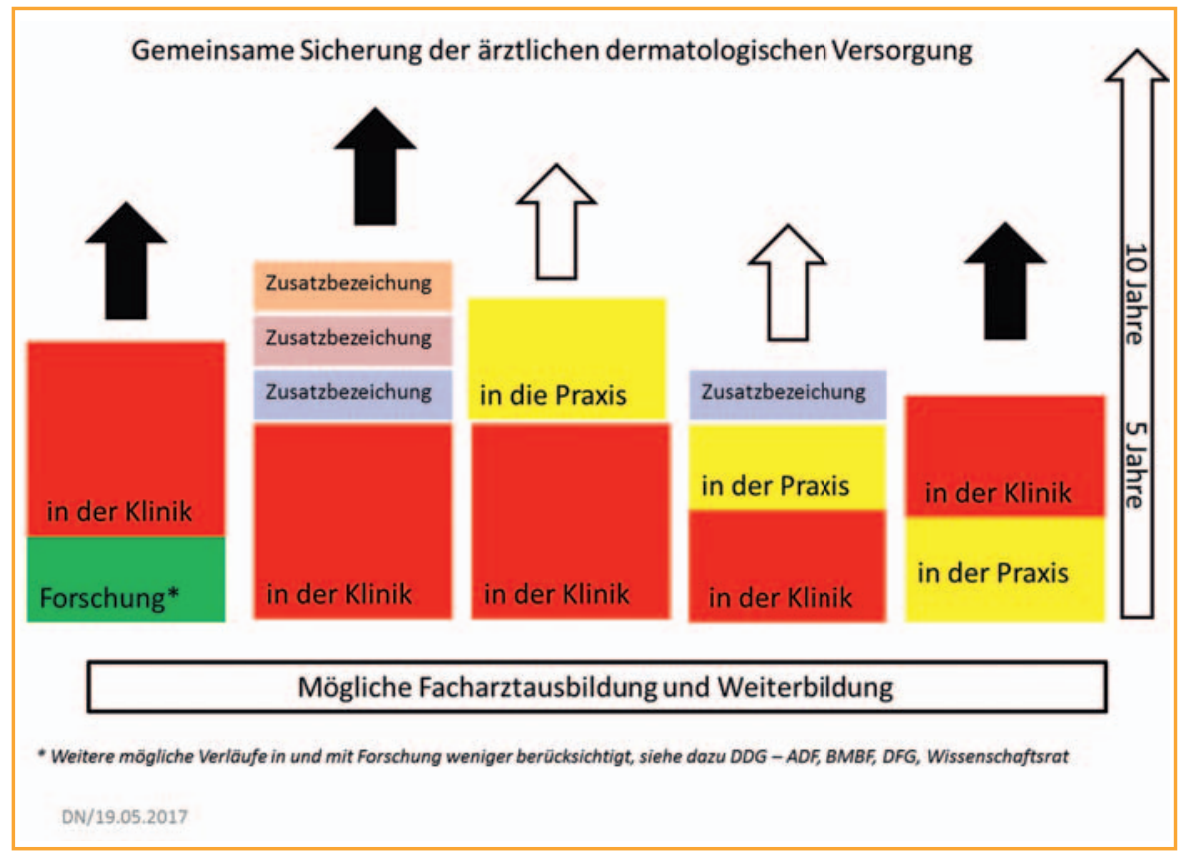

Abb. 1. Gemeinsame Sicherung der ärztlichen dermatologischen Versorgung. Ohne Anspruch auf Vollständigkeit sind hier mögliche Ausbildungssäulen dokumentiert mit einem mindestens 2,5-jährigen Einsatz im stationären Bereich, vielfach gepaart mit der Ausbildung in einer Praxis und anschließenden Ausbildungszeiten für mögliche Zusatzqualifikationen wie Allergologie, Phlebologie und medikamentöser Tumortherapie. ressen noch das Überstrapazieren in der beruflichen Einsatzplanung dient der persönlichen Entfaltung.

Sind diese Grundlagen geschaffen, können sich Berufsentwicklung und Karriere anschließen. Die Bandbreite der beruflichen Ausbildung ist in der Abbildung unter Berücksichtigung einer 5-jährigen Ausbildungszeit zum Facharzt für Dermatologie und Venerologie skizziert, u.a. mit Blick auf die bisher geltende Zeitspanne von obligaten 2,5 Jahren im stationären Einsatz (Abb. 1). Ärztekammern möchten mit flexibleren Weiterbildungszeiten ebenfalls die Organisation erleichtern; hier können auch individuelle Forschungszeiten im Rahmen der Weiterbildungszeit beantragt werden [1, 2].

Eigeninitiative ergreifen, Kompetenzen entwickeln, Wertschätzung entgegenbringen

Mit entsprechender Eigeninitiative und der Entwicklung von Kompetenzen steht der Mehrwert für Arbeitnehmer und Arbeitgeber außer Frage.

Der Arztberuf wird mit einem hohen menschlichen und zeitlichen Einsatz definiert, gepaart mit zahlreichen administrativen Aufgaben: Das verdient Wertschätzung! Für das Erlernen von administrativen Fähigkeiten, für die Strukturierung von komplexen Aufgaben und Einsatzzeiten sowie für die Entwicklung von Berufsstrategien neben der Familie sind die derzeitigen Strukturen zweifellos optimierbar. Dennoch gilt: Fürsorge erfüllt mit Glück, Mitgestalten macht Freude, und Lernen und Denken schaffen Freiheiten!

Literatur

1 Wenning $\mathrm{M}$, Van Aken H: Weiterbildung - Forschungstätigkeit und klinische Weiterbildung. Anasthesiol Intensivmed Notfallmed Schmerzther 2016;51:488-489.

2 Wenning M: Forschung in der Weiterbildung - ein Modellprojekt. Karger Kompass Ophthalmol 2017; 3:102-107.

Kontaktadresse: Prof. Dr. Dorothee Nashan, Hautklinik des Klinikum Dortmund gGmbH, Beurhausstraße 40, 44137 Dortmund, Deutschland, Dorothee.Nashan@klinikumdo.de. 


\section{Buchrezension}

Michael Sabel: Time and Life Management for Medical Students and Residents

Stuttgart, Thieme, 2017, 76 Seiten, 24,99 EUR

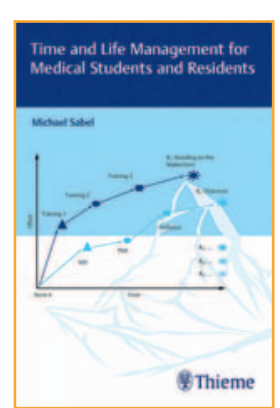

Das Buch von Herrn Sabel beschäftigt sich mit einem Thema, das im Medizinstudium leider keinen Platz gefunden hat und doch sehr wichtig ist. Die zentrale Frage lautet: Wie lerne ich mich selbst zu organisieren und meine Ziele zu erreichen?

Das Buch beginnt mit der Aufforderung, sich erst einmal über seine Ziele und Ambitionen klar zu werden. Hierzu werden verschiedene kleine Übungen und Denkex-

perimente vorgeschlagen. Das Buch wird erst dann zu einer wertvollen Hilfe, wenn diese Übungen auch tatsächlich durchgeführt werden, denn alle folgenden Kapitel setzen klar gesteckte Ziele voraus. Ist man sich über seine Ziele bewusst geworden, so geht es im zweiten Abschnitt darum, wie man diese erreichen kann. Wie kann ich mich motivieren meine Ziele zu verfolgen und nicht aufzugeben? Wie entscheide ich, welche Aufgaben wirklich wichtig sind? Es geht in diesem Buch um das, was wohl die meisten Medizinstudenten nach dem Studium qualvoll lernen müssen: den Alltag auf Station effektiv zu gestalten, sich auch einmal abzugrenzen und die nötige Balance zwischen Arbeits- und Privatleben zu finden. Konkrete Vorschläge, wie man seine Effektivität dann tatsäch- lich erhöhen kann, liefert das Buch meiner Meinung nach leider nur eingeschränkt, bleibt es doch häufig bei allgemeinen Grundsätzen. Die vielen Graphiken verdeutlichen die im Text beschriebenen Theorien sehr plastisch, liefern aber keine neue Aussage und waren mir zu trivial. Für Menschen, die sich bereits mit dem Thema «Selbstmanagement» beschäftigt haben, mag das Buch augenscheinlich keine neuen Erkenntnisse bieten. Außerdem fehlte mir ein stringenter Aufbau. Alle Kapitel waren zwar in sich schlüssig aufgebaut, jedoch fehlte der übergeordnete Zusammenhang zwischen den Kapiteln.

Trotz allem hat das Buch seine Daseinsberechtigung, denn eines tut es sicher: Es regt zum Denken an. Was für ein Arzt möchte ich sein? Was für ein Mensch möchte ich sein? Wo liegen meine Prioritäten? Sind meine Ziele realistisch und nutze ich meine Zeit sinnvoll? Sich bereits vor Arbeitsbeginn einmal mit diesen Fragen auseinanderzusetzen spart in der ohnehin stressigen Anfangszeit sicherlich viel Zeit und Kraft.

Clara Vollmer, Kiel

Kontaktadresse: Clara Vollmer, Klinik für Innere Medizin IV, Nieren- und Hochdruckkrankheiten, Universitätsklinikum Schleswig-Holstein, Schittenhelmstraße 12, 24105 Kiel, Deutschland, claracvollmer @ googlemail.com. 